\title{
EFEITOS DA ERITROPOETINA RECOMBINANTE HUMANA EM RECÉM-NASCIDOS PRÉ-TERMO COM DOENÇAS INFECCIOSAS
}

\author{
Iara flávia de Vasconcelos P. Aguiar*, Vera lúcia Jornada Krebs, Sandra f. M. Gualandro, Paulo A. A. Silveira, flávio Adolfo Costa Vaz \\ Trabalho realizado na Unidade de Cuidados Intensivos Neonatal do Instituto da Criança do Hospital das Clínicas da Faculdade de Medicina da
} Universidade de São Paulo

\author{
*Correspondência: \\ Rua Pedro Pomponazzi, \\ 890 , apto 61 \\ Chácara Klabin \\ São Paulo/SP \\ Cep: $04115-000$ \\ Tel: (11) 5539-6024 \\ iaraflavia@uol.com.br
}

\begin{abstract}
RESUMO
OBJEtivo. Analisar os efeitos da eritropoetina recombinante humana (rHuEpo) em recém-nascidos pré-termo com doenças infecciosas graves.

Métodos. Foi realizado um estudo controlado, não randomizado, em 34 recém-nascidos com diagnóstico de patologias infecciosas graves, peso de nascimento igual ou inferior a 1500 g, idade gestacional inferior a 35 semanas e estabilidade clínica. Os recém-nascidos designados para o tratamento com rHuEpo receberam a eritropoetina $B$ na dose de $400 \mathrm{UI} / \mathrm{kg}$, duas vezes por semana, por via subcutânea. A suplementação oral com ferro foi iniciada quando os níveis de ferritina sérica foram inferiores a $60 \mathrm{mcg} / \mathrm{L}$. $O$ estudo foi realizado durante seis semanas ou até a alta hospitalar do paciente. Foram avaliados a eritropoese, o número de transfusões, o número de neutrófilos, a contagem de plaquetas e os episódios de novas infecções durante 0 tratamento com o hormônio.

Resultados. Houve aumento significativo do número de reticulócitos no grupo tratado; entretanto, não houve impacto sobre o número ou volume de transfusões. Não foram observadas alterações no número de neutrófilos ou plaquetas.

Conclusä́o. $\mathrm{O}$ uso de rHuEpo em RNPT com doenças infecciosas, na dose de $800 \mathrm{UI} / \mathrm{Kg} / \mathrm{semana}$, foi efetivo para induzir eritropoese, sem ocorrerem alterações significativas sobre o número de neutrófilos ou plaquetas. Essa estratégia, associada ao controle rigoroso do volume de sangue retirado para exames, poderá ser benéfica na prevenção da anemia em RNPT com infecção grave.
\end{abstract}

UNITERMOS: Anemia neonatal. Infecção neonatal. Eritropoetina recombinante. Recém-nascido.

\section{INTRODUÇÃo}

Apesar da eritropoetina recombinante humana (rHuEpo) ter sido utilizada no tratamento da anemia da prematuridade, poucos estudos utilizaram este hormônio para o tratamento de recém-nascidos prétermo (RNPT) doentes'. A infecção pode provocar hemólise e depressão da ação da eritropoetina, podendo causar anemia nesses RNPT, que também necessitam de constantes coletas de sangue para exames laboratoriais. Para diminuir a necessidade de transfusões sangüíneas, o uso de rHuEpo poderia ser benéfico nestas crianças. O objetivo do presente estudo é analisar os efeitos da eritropoetina recombinante humana em recém-nascidos pré-termo com doenças infecciosas graves, internados em Unidade de Terapia Intensiva Neonatal.

\section{Métodos}

Foi realizado um estudo controlado, não-randomizado, em 34 recém-nascidos pré-termo com diagnóstico de patologias infecciosas graves, internados na Unidade de Cuidados Intensivos Neonatal do Instituto da Criança do Hospital das Clínicas da Faculdade de Medicina da Universidade de São Paulo. Vinte RNPT que receberam a rHuEpo constituíram o grupo de estudo, e 14 que não foram tratados com o hormônio participaram do grupo controle. Foram incluídos na casuística os recém-nascidos com as seguintes características: idade gestacional < 35 semanas; idade pós-natal até 28 dias; peso de nascimento < I 500 g; estabilidade clínica (ausência de distúrbio ácidobásico e eletrolítico; oxigenação adequada, mesmo se em ventilação mecânica; ausência de convulsões e hipertensão arterial); presença de doenças infecciosas graves (sepse, meningite, pneumonia, onfalite, pioartrite).

Foram excluídos os RN portadores de neutropenia (neutrófilos < $500 / \mathrm{mm}^{3}$ nas primeiras 60 horas de vida ou neutrófilos $<1100 / \mathrm{mm}^{3}$ após 60 horas de vida ${ }^{2}$ ), malformações congênitas graves, cardiopatias complexas, hemorragia intracraniana grau III ou IV, doença hemolítica causada por deficiência de G6PD, incompatibilidade $A B O$ ou Rh, convulsões, necessidade de cirurgia e valores de $\mathrm{Ht}>49 \%$.

Os recém-nascidos designados para o tratamento com rHuEpo receberam a eritropoetina B na dose de $400 \mathrm{UI} / \mathrm{kg}$, duas vezes por semana, por via subcutânea, na face anterior da perna direita. A suplementação oral com ferro foi iniciada quando os níveis de ferritina sérica foram inferiores a $60 \mathrm{mcg} / \mathrm{L}$. A dose de ferro foi de $4 \mathrm{mg} / \mathrm{kg} / \mathrm{dia}$ de ferro elementar. $O$ estudo foi realizado durante seis semanas. Quando a alta hospitalar ocorreu antes deste período, a última amostra foi colhida no momento desta.

Foram avaliados a eritropoese, o impacto do uso de eritropoetina recombinante humana sobre o número de transfusões e o volume de sangue transfundido. Foram também estudados o número de 
Tabela I - Valores médios de $\mathrm{Hb}$, $\mathrm{Ht}$, reticulócitos, leucócitos, neutrófilos e plaquetas no grupo tratado e no grupo controle de recém-nascidos pré-termo com doenças infecciosas

\begin{tabular}{|c|c|c|c|c|}
\hline & Dia do estudo & Grupo tratado & Grupo controle & $\mathrm{p}$ \\
\hline $\mathrm{Hb}(\mathrm{g} / \mathrm{dl})$ & $\begin{array}{c}1^{\circ} \\
7^{\circ} \\
21^{\circ} \\
F \\
\end{array}$ & $\begin{array}{l}13,64 \\
12,31 \\
10,93 \\
9,29 \\
\end{array}$ & $\begin{array}{c}|3,4| \\
\mid 1,47 \\
10,02 \\
9,81 \\
\end{array}$ & $\begin{array}{l}0,739 \\
0,184 \\
0,087 \\
0,466\end{array}$ \\
\hline $\mathrm{Ht}(\%)$ & $\begin{array}{c}1^{\circ} \\
7^{\circ} \\
21^{\circ} \\
F \\
\end{array}$ & $\begin{array}{l}37,80 \\
34,10 \\
30,80 \\
26,70\end{array}$ & $\begin{array}{l}37,50 \\
32,10 \\
28,20 \\
27,90\end{array}$ & $\begin{array}{l}0,903 \\
0,248 \\
0,094 \\
0,496\end{array}$ \\
\hline Reticulócitos(\%) & $\begin{array}{c}1^{\circ} \\
7^{\circ} \\
21^{\circ} \\
F\end{array}$ & $\begin{array}{l}4,23 \\
5,12 \\
4,91 \\
5,26\end{array}$ & $\begin{array}{l}3,93 \\
2,77 \\
2,82 \\
2,73\end{array}$ & $\begin{array}{l}0,779 \\
0,007 \\
0,018 \\
0,016\end{array}$ \\
\hline Leucócitos(células/mm³) & $\begin{array}{c}1^{\circ} \\
7^{\circ} \\
21^{\circ} \\
F\end{array}$ & $\begin{array}{l}12220 \\
15620 \\
11396 \\
10013 \\
\end{array}$ & $\begin{array}{l}11957 \\
11986 \\
10097 \\
9804 \\
\end{array}$ & $\begin{array}{l}0,865 \\
0,029 \\
0,467 \\
0,604 \\
\end{array}$ \\
\hline Neutrófilos (células/mm³) & $\begin{array}{c}1^{\circ} \\
7^{\circ} \\
21^{\circ} \\
F \\
\end{array}$ & $\begin{array}{l}5424 \\
6954 \\
4036 \\
3238 \\
\end{array}$ & $\begin{array}{l}5648 \\
4377 \\
3096 \\
2160 \\
\end{array}$ & $\begin{array}{l}0,841 \\
0,064 \\
0,458 \\
0,203 \\
\end{array}$ \\
\hline Plaquetas(células/mm³) & $\begin{array}{c}1^{\circ} \\
7^{\circ} \\
21^{\circ} \\
F\end{array}$ & $\begin{array}{l}280900 \\
353400 \\
327842 \\
336200\end{array}$ & $\begin{array}{l}260143 \\
31457 \mid \\
265857 \\
319182\end{array}$ & $\begin{array}{l}0,683 \\
0,517 \\
0,296 \\
0,594\end{array}$ \\
\hline
\end{tabular}

F: final

neutrófilos, a contagem de plaquetas e os episódios de novas infecções durante o tratamento com o hormônio.

Foram realizados os seguintes exames: nível sérico de eritropoetina e nível sérico de ferritina (ao início, com três semanas e ao término do estudo); hemograma, contagem de plaquetas e reticulócitos (ao início, no sétimo dia, no final da terceira semana e ao término do estudo).

O volume de sangue retirado para amostras, o número e volume de transfusões administradas, o peso e o aporte hídrico, calórico e protéico foram registrados diariamente. As transfusões sangüíneas foram indicadas pelo médico que atendia o recém-nascido na UTI neonatal, conforme a norma do serviço.

Para comparar as médias dos valores de $\mathrm{Hb}$, $\mathrm{Ht}$, reticulócitos, leucócitos, neutrófilos, plaquetas, ferritina, ferro sérico, eritropoetina sérica e número de transfusões foi utilizado o teste de t não emparelhado. Para comparar o volume de transfusões foi utilizado o teste de Mann-Whitney. Para comparar as características clínicas do grupo tratado e do grupo controle foram utilizados o teste do Qui-quadrado e o teste exato de Fisher. Foram considerados significativos os valores de $p$ inferiores a 0,05.

O estudo foi aprovado pela Comissão de Ética Institucional. Para todos os pacientes, foi obtido o termo de consentimento livre $\mathrm{e}$ esclarecido, com a assinatura dos pais.

\section{Resultados}

Ao início do estudo, ambos os grupos foram semelhantes em relação ao peso de nascimento $(p=0,629)$, idade gestacional $(p=0,276)$, idade pós-natal $(p=0,757)$ e distribuição entre os sexos $(p=1,00)$. A sepse foi o diagnóstico mais freqüente, ocorrendo em 30 recém-nascidos. Outras infecções foram onfalite e broncopneumonia.

A duração do estudo variou de 23 a 43 dias no grupo tratado (média=38), e de 19 a 43 dias no grupo controle (média =37 dias). Dez (50\%) recém-nascidos do grupo tratado e oito $(57,1 \%)$ do grupo controle necessitaram de ventilação pulmonar mecânica durante 0 período de estudo $(p=0,738)$. Os valores médios de $\mathrm{Hb}, \mathrm{Ht}$, reticulócitos, leucócitos, neutrófilos e plaquetas nos dois grupos estão apresentados na Tabela I.

Observou-se neutropenia (neutróflos $<500 / \mathrm{mm}^{3}$ nas primeiras 60 horas de vida ou neutrófilos $<1100 / \mathrm{mm}^{3}$ após 60 horas de vida) em seis RN (42,86\%) do grupo controle e quatro RN (20\%) do grupo tratado. Nesses pacientes, a administração de rHuEpo foi suspensa.

Durante o período de estudo, nove RN (45\%) do grupo tratado apresentaram novos episódios de infecção; em seis destes a hemocultura mostrou: Acinetobacter baumanni, Klebsiella oxytoca, Enterococcusfaecalis, Staphylococcus epidermidis, Burkholderia cepacea e Candida sp. No grupo controle, seis RN (42,8\%) apresentaram novas infecções. Na hemocultura, houve crescimento de Enterobacter 


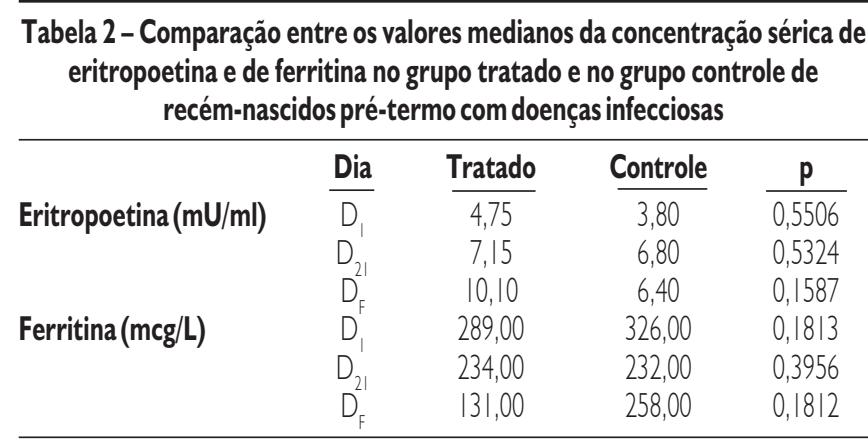

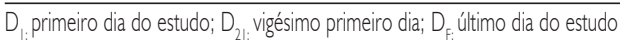

agglomerans, Enterococcus faecalis, Staphylococcus epidermidis, Staphylococcus coagulase-negativa, Serratia marcenscens e Candida albicans. Um RN apresentou duas uroculturas com crescimento de Citrobacter diversus. A ocorrência de novos episódios de infecção não diferiu entre os grupos $(p=1,000)$.

Os níveis séricos de ferritina e de eritropoetina estão apresentados na Tabela 2. Três RN tratados apresentaram ferritina inferior a 60 mcg/ $L$ no $21^{\circ}$ dia de estudo e ao final do estudo, fato observado em apenas um RN do grupo controle. A suplementação de ferro foi introduzida em todos estes RN, exceto em um RN tratado que havia recebido transfusão após a coleta.

Com relação ao número total de transfusões, cinco recém-nascidos (35,7\%) do grupo controle e cinco recém-nascidos (25\%) do grupo tratado haviam recebido transfusões de concentrado de hemácias antes do início do estudo. Durante o período de estudo, o número médio de transfusões de sangue no grupo tratado foi de I,45, com variação de 0 a 7 transfusões; no grupo controle, o número médio foi de 2,36, com variação de 0 a 9 transfusões $(p=0,270)$. $O$ volume médio de sangue transfundido no grupo tratado, no período avaliado, foi de $16,53 \mathrm{ml} / \mathrm{kg}$, com variação de 0 a $74,18 \mathrm{ml} / \mathrm{kg}$, enquanto o grupo controle recebeu o volume médio de $26,01 \mathrm{ml} / \mathrm{kg}$, com variação de 0 a $97,87 \mathrm{ml} / \mathrm{kg}(p=0,325)$.

aporte hídrico, calórico e protéico foi semelhante nos dois grupos durante todo o estudo, com exceção da última semana, quando o grupo tratado recebeu oferta protéica inferior à do grupo controle $(p=0,026)$. O grupo tratado recebeu oferta hídrica variável de I52,32 a | 65,23 ml/kg/dia, oferta calórica variável de 80,35 a I | 5,22 cal/kg/dia e oferta protéica variável de 2,37 a 2,67 g/kg/dia. Não houve diferenças significativas entre os grupos em relação ao ganho de peso, com variação de 255 g a 1055 g (média=648 g) no grupo tratado, e de 355 g a $920 \mathrm{~g}$ (média $=649,28 \mathrm{~g}$ ) no grupo controle $(\mathrm{p}=0,983)$.

\section{Discussão}

A análise dos dois grupos não mostrou diferença significativa em relação ao peso de nascimento, idade gestacional, idade ao início do estudo, distribuição entre os sexos, valores hematológicos, ferritina e eritropoetina séricas, podendo ambos serem considerados semelhantes com respeito a estas variáveis. Embora o grupo controle tenha mostrado as mesmas características do grupo que recebeu rHuEpo, a ausência de randomização pode ter influenciado a distribuição de fatores não controláveis entre os grupos, constituindo uma limitação do nosso estudo.
Houve aumento significativo do número de reticulócitos no grupo tratado com rHuEpo, desde a primeira semana até o final do estudo, sugerindo que houve indução da eritropoese. Apesar disso, não houve diferença significativa entre os valores de $\mathrm{Hb}$ e de $\mathrm{Ht}$ entre os grupos, ou da necessidade de transfusões sangüíneas. Este comportamento difere de resultados obtidos por outros autores, que mostraram aumento significativo da $\mathrm{Hb}$ e do Ht após a administração de rHuEpo em recém-nascidos pré-termo ${ }^{3-11}$. No entanto, a casuística destas pesquisas foi composta, na sua maioria, por recém-nascidos pré-termo não infectados, ao contrário do nosso estudo, que analisou neonatos portadores de doenças infecciosas graves.

Sabe-se que as infecções diminuem a eritropoese em adultos. Em recém-nascidos, Brown e Keith'2, observaram diminuição da eritropoese na presença de sepse e enterocolite necrosante durante o tratamento com rHuEpo, sugerindo haver refratariedade ao hormônio na presença de infecção. Segundo esses autores, a ação de algumas citocinas, como a interleucina 6 (IL-6) e o fator de necrose tumoral- $\alpha$ (TNF $\alpha$ ), presentes na sepse e enterocolite necrosante, pode concorrer para deprimir a eritropoese. Seeherunvong et al. ${ }^{13}$ sugerem que a ausência de resposta à rHuEpo durante a inflamação deva-se ao aumento da produção de citocinas, que podem induzir resistência de células progenitoras eritróides ao hormônio, ou à disponibilidade limitada de ferro do estoque reticuloendotelial para a produção do heme.

$\mathrm{O}$ aporte de substratos para a formação da $\mathrm{Hb}$ é fundamental para que se atinja a resposta eritropoética máxima. A eritropoese é dependente de nutrição adequada e, principalmente, da oferta protéica e de ferro. Tal dependência é particularmente pronunciada durante o rápido crescimento observado em RNPT ${ }^{14}$. A oferta protéica deve ser suficiente para permitir eritropoese e garantir o rápido crescimento corpóreo do RNPT, sem risco de que o estímulo com o hormônio exógeno desvie proteínas para a produção de células vermelhas, com prejuízo do crescimento essencial.

Bechensteen et al. ${ }^{15,16}$, apesar de não utilizarem doses elevadas de rHuEpo, observaram efeito estimulatório do hormônio, fornecendo altas doses de ferro e proteínas na quantidade de $3 \mathrm{~g} / \mathrm{Kg} / \mathrm{dia}$. Os RN tratados em nosso estudo receberam, em média, 2,5 g/Kg/dia a 2,6 g/ $\mathrm{Kg} /$ dia de proteínas, do início ao término do estudo, e oferta calórica média de 80 a 109,7 cal/Kg/dia. A presença de patologias infecciosas graves nestes $\mathrm{RN}$ constituiu um fator limitante do aumento rápido da oferta de nutrientes. É possível que o aporte protéico-calórico tenha sido insuficiente para sustentar a formação de glóbulos vermelhos.

A suplementação de ferro foi efetuada em apenas dois recémnascidos do nosso estudo, cujos valores de ferritina foram inferiores a $60 \mathrm{mcg} / \mathrm{L}$. Na maioria dos estudos randomizados e controlados sobre o uso de rHuEpo em recém-nascidos pré-termo, o ferro foi administrado por via oral ou endovenosa, em doses variáveis de I a $10 \mathrm{mg} / \mathrm{Kg} /$ dia, 3,5-11,17-26. Estudos anteriores demonstraram associação entre o uso de ferro e maior suscetibilidade para sepse neonatal por bactérias gram-negativas ${ }^{27,28}$.

Apesar desses achados estarem relacionados ao uso de ferro dextran, administrado por via intramuscular, trouxe-nos preocupação o uso indiscriminado de ferro para RNPT infectados, já que o ferro age como doador de elétron na formação de radicais livres. $\bigcirc$ ferro livre, 
originado de transfusões de concentrado de hemácias ou da suplementação com sulfato ferroso, poderia constituir um fator predisponente para a ocorrência de lesão tecidual oxidativa em RNPT de muito baixo peso ao nascimento, submetidos à intensa exposição ao oxigênio e apresentando sistemas de defesa antioxidante endógeno imaturos $^{29}$.

Em nossa casuística utilizamos o critério de administrar ferro quando os neonatos apresentassem níveis baixos de ferritina sérica, que constitui bom indicador das reservas de ferro e permite detectar uma carência absoluta deste elemento. No RN, os valores normais da ferritina sérica são extremamente variáveis, sendo níveis inferiores a $65-100 \mathrm{mcg} / \mathrm{L}$ sugestivos de carência de ferro estocado ${ }^{30}$. Em crianças com eritropoese estimulada pela rHuEpo, são usados valores de corte de ferritina plasmática de 60 a $100 \mathrm{mcg} / \mathrm{L}^{31}$. No entanto, as concentrações de ferritina não se correlacionam apenas com o status do ferro. Entre as situações que podem modificar seus níveis plasmáticos, incluem-se os processos infecciosos, especialmente as infecções crônicas. Nestas condições, os níveis plasmáticos de ferritina aumentam, provavelmente devido à resposta inflamatória associada. Em nossa casuística, durante todo o período do estudo, a concentração mediana de ferritina foi superior a $100 \mathrm{mcg} / \mathrm{L}$. É possível que, na ausência de processo infeccioso, estes valores fossem mais baixos, tanto no grupo tratado como no grupo controle. Dimitriou et al. ${ }^{32}$ recomendam o cálculo do índice "receptor solúvel de transferrina/log ferritina" para a identificação da deficiência de ferro em crianças com infecção concomitante.

$\mathrm{Na}$ comparação intragrupo dos níveis séricos de eritropoetina, observamos aumento significativo no grupo tratado, desde o início até o final do estudo, fato não observado no grupo controle, conforme era esperado. Na comparação entre os dois grupos, observamos aumento da eritropoetina no grupo tratado, embora sem diferença significativa. É possível que doses maiores de eritropoetina ou intervalos mais curtos entre as doses resultassem em níveis séricos mais altos.

A ocorrência de neutropenia e a contagem de plaquetas foi semeIhante entre os grupos tratado e controle. Embora estudos iniciais, sem grupo controle, tenham sugerido a possibilidade de neutropenia em recém-nascidos tratados com $\mathrm{rHuEpo}^{33}$, estudos multicêntricos randomizados e controlados, com casuística numerosa, não observaram este efeito6,18. Meister et al." demonstraram que não houve evidência de desvio das células multipotentes (stem cells) para a formação de precursores eritróides, em detrimento de precursores mielóides.

\section{Conclusão}

O estudo mostra que o uso de rHuEpo em RNPT com doenças infecciosas, na dose de $800 \mathrm{UI} / \mathrm{Kg} / \mathrm{semana}$, resultou no aumento do número de reticulócitos, sugerindo que houve indução da eritropoese, sem ocorrerem alterações significativas sobre o número de neutrófilos ou plaquetas. A utilização de rHuEpo poderá ser benéfica na prevenção da anemia em RNPT com infecção grave.

No entanto, o número de recém-nascidos avaliados foi pequeno, sendo recomendada a realização de novos estudos multicêntricos, com maior tamanho amostral.

\section{Conflito de interesse: não há.}

\section{SUMMARY \\ EFFECTS OF RECOMBINANT hUMAN ERYTHROPOIETIN IN PRETERM NEWBORNS WITH INFECTIOUS DISEASES}

OBIECTNE. To study the effects of recombinant human erythropoietin (rHuEpo) in preterm newborns (PTNs) with serious infectious diseases.

METHODS. Anot randomized case-control study was carried out in 34 preterm newborns with diagnosis of serious infectious pathologies, gestational age up to 35 weeks, birth weight less than I $500 \mathrm{~g}$ and clinical stability. Newborns selected for treatment with rHuEpo received $400 \mathrm{U} /$ kg erythropoietin B, subcutaneously twice a week. Oral iron supplementation was initiated when the levels of serum ferritin were lower than 60 $m c g /$. The study was continued for six weeks or until the patient was discharged from the hospital. Erythropoiesis, granulopoiesis, thrombocytopoiesis, the need for transfusions and the occurrence of new episodes of infectious disease were analyzed.

RESULTS. In the treated group there was a significant increase in the number of reticulocytes, although there was no statistically significant difference between the groups with regard to the number or volume of transfusions. There was no significant difference in neutrophils and platelet values.

Conclusion. The use ofrHuEpo, $800 \mathrm{U} / \mathrm{kg} /$ week, in PTNswithinfectious diseases was effective in inducing erythropoiesis, without significant changes in the number of neutrophils or platelets. This strategy, and the accurate control of the blood collected for laboratory exams, may be beneficial for prevention of the anemia in PTNs with serious infectious diseases. [Rev Assoc Med Bras 2007; 53(I): 90-4]

KEY WORDS: Neonatal anemia. Diseases of the preterm newborn. Recombinant erythropoietin. Neonate.

\section{REFERÊNCIAS}

I. Ohls RK, Ehrenkranz RA, Wright LL, Lemons JA, Korones SB, Stoll BJ, et al. Effects of early erythropoietin therapy on the transfusion requirements of preterm infants below 1250 grams birth weight: a multicenter, randomized, controlled trial. Pediatrics. 2001;108:934-42.

2. Mouzinho A, Rosenfeld CR, Sánchez PJ, Risser R. Revised reference ranges for circulating neutrophils in very-low-birth-weight neonates. Pediatrics. 1994;94:76-82.

3. Shannon KM, Mentzer WC, Abels R, Wertz M, Moriyama J, Li W, et al. Enhancement of erythropoiesis by recombinant human erythropoietin in low birth weight infants: a pilot study. J Pediatr. 1992; 1 20:586-92.

4. Carnielli V, Montini G, Da Riol R, Dall'amico R, Cantarutti F. Effect of high doses of human recombinant erythropoietin on the need for blood transfusions in preterm infants. J Pediatr. 1992; 121:98-102.

5. Meyer MP, Meyer JH, Commerford A, Hann FM, Sive AA, Moller G, et al. Recombinant human erythropoietin in the treatment of the anemia of prematurity: results of a double-blind, placebo-controlled study. Pediatrics. 1994;93:918-23.

6. Shannon KM, Keith JF, Mentzer WC, Ehrenkranz RA, Brown MS, Widness JA, et al. Recombinant human erythropoietin stimulates erythropoiesis and reduces erythrocyte transfusions in very low birth weight preterm infants. Pediatrics. 1995;95: I-8.

7. 7.Soubasi V, Kremenopoulos G, Diamandi E, Tsantali C, Arafidis K, Tsakiris $D$. Follow-up of very low birth weight infants after erythropoietin treatment to prevent anemia of prematurity. J Pediatr. 1995; 127:291-7.

8. Bader D, Blondheim O, Jonas R, Admoni O, Abend-Winger M, Reich D, et al. Decreased ferritin levels, despite iron supplementation, during erythropoietin therapy in anaemia of prematurity. Acta Paediatr. |996;85:496-50|. 
9. Samanci N, Ovali F, Dagoglu T. Effects of recombinant human erythropoietin in infants with very low birth weights. J Int Med Res. 1996;24:190-8.

I0. Manchon GJ, Pujol A, Lletget W, Cotro A, Barnusell J, Piera J, et al. Estudio multicéntrico aleatorizado de administración de eritropoyetina en la anemia de la prematuridad. An Esp Pediatr. 1997;46:587-92.

I I. Meister B, Maurer H, Simma B, Kern H, Ulmer H, Hittmair A, et al. The effect of recombinant human erythropoietin on circulating hematopoietic progenitor cells in anemic premature infants. Stem Cells. 1997; 1 5:35963.

12. Brown MS, Keith JF. Comparison between two and five doses a week of recombinant human erythropoietin for anemia of prematurity: a randomized trial. Pediatrics. 1999; 104:210-5.

13. Seeherunvong W, Rubio L, Abitbol CL, Montané B, Strauss J, Diaz R, et al. Indentification of poor responders to erythropoietin among children undergoing hemodialisis. J Pediatr. 200 I; 138:7 I0-4.

14. Bechensteen AG, Halvorsen S, Haga P, Cotes PM, lestol K. Erythropoietin (epo), protein and iron supplementation and the prevention of anaemia of prematurity: effects on serum immunoreactive epo, growth and protein and iron metabolism. Acta Paediatr. 1996;85:490-5.

I5. Bechensteen AG, Haga P, Halvorsen S, Whitelaw A, Liestol K, Lindemann R. Erythropoietin, protein, and iron supplementation and the prevention of anaemia of prematurity. Arch Dis Child. 1993;69:19-23.

16. Bechensteen AH, Haga P, Halvorsen S, Liestol K, Lindemann R, Whitelaw PA, et al. Effect of low and moderate doses of recombinant human erythropoietin on the haematological response in premature infants on a high protein and iron intake. Eur J Pediatr. 1997; | 56:56-6I.

17. Ohls RK, Christensen RD. Recombinant erythropoietin compared with erythrocite transfusion in the treatment of anemia of prematurity. J Pediatr. 1991; 1 19:781-8.

18. Maier RF, Obladen M, Scigalla P, Linderkamp O, Duc G, Hieronimi G, et al. The effect of epoetin beta (recombinant human erythropoietin) on the need for transfusion in very-low-birth-weight infants. N Engl J Med. 1994;30: 1 173-8.

19. Bock W, Gröhs B, Minihold B, Schemper M, Lischka A. Comparison of high dose therapy of rhepo, given two or three times a week in premature infants. J Perinat Med. 1995;23: I33-8.

20. Ohls RK, Osborne KA, Christensen RD. Efficacy and cost analysis of treating very low birth weight infants with erythropoietin during their first two weeks of life: a randomized, placebo-controlled trial. J Pediatr. 1995; | 26:421-6.

21. Ohls RK, Veerman MW, Christensen RD. Pharmacokinetics and effectiveness of recombinant erythropoietin administered to preterm infants by continuous infusion in total parenteral nutrition solution. J Pediatr. 1996; 1 28:518-23.

22. Griffiths G, Lall R, Chatfield S, Short A, Mackay P, Williamson P, et al. Randomised controlled double blind study of role of recombinant erythropoietin in the prevention of chronic lung disease. Arch Dis Child. 1997;76:F190-2.
23. Maier RF, Obladen M, Kattner E, Natzschka J, Messer J, Regazzoni B, et al. High-versus low-dose erythropoietin in extremely low birth weight infants. J Pediatr. 1998; 132:866-70.

24. Kumar P, Shankaran S, Krishnan R. Recombinant human erythropoietin therapy for treatment of anemia of prematurity in very low birth weight infants: a randomized, double-blind, placebo-controlled trial. J Perinatol. 1998; | 8:173-7.

25. Giannakopoulou C, Bolonaki I, Stiakaki E, Dimitriou H, Galanaki H, Hatzidaki E, et al. Erythropoietin (rhuepo) administration to premature infants for the treatment of their anemia. Pediatr Hematol Oncol. 1998; 15;37-43.

26. Ohls RK, Harcum J, Schibler K, Christensen R. The effect of erythropoietin on the transfusion requirements of preterm infants weighing 750 grams or less: a randomized, double-blind, placebo-controlled study. J Pediatr. 1997; |31:66|-5.

27. Barry DMJ, Reeve AW. Increased incidence of gram-negative neonatal sepsis with intramuscular iron administration. Pediatrics. 1977;60:908-12.

28. Becroft DMO, Dix MR, Farmer K. Intramuscular iron-dextran and susceptibility of neonates to bacterial infections. Arch Dis Child. 1977;52:778-8I.

29. Pollak A, Hayde M, Hayn M, Herkner K, Lombard KA, Lubec G, et al. Effect of intravenous iron supplementation on erythropoiesis in erythropoietin-treated premature infants. Pediatrics. 2001; 107:78-85.

30. Picaud JC, Putet G, Salle BL, Claris O. Supplémentation en fer chez les enfants prématurés traités par erythropoïétine. Arch Pediatr. 1999;6:657-64

31. Kling PJ, Winzerling JJ. Iron status and the treatment of the anemia of prematurity. Clin Perinatol. 2002;29:283-94.

32. Dimitriou H, Stiakaki E, Markaki EA, Bolonaki I, Giannakopoulou CH, Kalmanti M. Soluble transferring receptor levels and soluble transferrin receptor/log ferritin index in the evaluation of erythropoietic status in childhood infections and malignancy. Acta Paediatr. 2000;89:1 169-73.

33. Halpérin DS, Wacker P, Lacourt G, Félix M, Babel JF, Aapro M, et al. Effects of recombinant human erythropoietin in infants with the anemia of prematurity: a pilot study. J Pediatr. 1990; | 16:779-86.

Artigo recebido: 06/02/06

Aceito para publicação: 26/10/06 